\title{
NEW SUBCLASSES OF THE CLASS OF CLOSE-TO-CONVEX FUNCTIONS
}

\author{
PRAN NATH CHICHRA
}

ABSTRact. In this paper we introduce new subclasses of the class of closeto-convex functions. We call a regular function $f(z)$ an alpha-close-to-convex function if $\left(f(z) f^{\prime}(z) / z\right) \neq 0$ for $z$ in $E$ and if for some nonnegative real number $\alpha$ there exists a starlike function $\phi(z)=z+\cdots$ such that

$$
\operatorname{Re}\left[(1-\alpha) \frac{z f^{\prime}(z)}{\phi(z)}+\alpha \frac{\left(z f^{\prime}(z)\right)^{\prime}}{\phi^{\prime}(z)}\right]>0
$$

for $z$ in $E$.

We have proved that all alpha-close-to-convex functions are close-toconvex and have obtained a few coefficient inequalities for $\alpha$-close-to-convex functions and an integral formula for constructing these functions.

Let $\mathcal{F}_{\alpha}$ be the class of regular and normalised functions $f(z)$ which satisfy $\operatorname{Re}\left(f^{\prime}(z)+\alpha z f^{\prime \prime}(z)\right)>0$ for $z$ in $E . f(z) \in \mathscr{F}_{\alpha}$ gives $\operatorname{Re} f^{\prime}(z)>0$ for $z$ in $E$ provided $\operatorname{Re} \alpha \geqslant 0$. A sharp radius of univalence of the class of functions $f(z)$ for which $z f^{\prime}(z) \in \mathscr{F}_{\alpha}$ has also been obtained.

1. Recently Mocanu [4] introduced the concept of alpha-starlike functions. He called a function $f(z)$ an $\alpha$-starlike function if $\left(f(z) f^{\prime}(z) / z\right) \neq 0$ in $E=\{z /|z|<1\}$ and if for some real number $\alpha$,

$$
\operatorname{Re}\left[(1-\alpha) \frac{z f^{\prime}(z)}{f(z)}+\alpha\left(1+\frac{z f^{\prime \prime}(z)}{f^{\prime}(z)}\right)\right]>0
$$

for $z$ in $E$. It is shown in [5] that all $\alpha$-starlike functions are starlike in $E$. Moreover for $\alpha \geqslant 1$, all $\alpha$-starlike functions are convex in $E$.

In this note we shall introduce new subclasses of the class of close-to-convex functions. We shall call $f(z)=z+a_{2} z^{2}+\cdots$ an alpha-close-to-convex function if $\left(f(z) f^{\prime}(z) / z\right) \neq 0$ in $E$ and if for some nonnegative real number $\alpha$ and for some starlike function $\phi(z)=z+\cdots$,

$$
\operatorname{Re}\left[(1-\alpha) \frac{z f^{\prime}(z)}{\phi(z)}+\alpha \frac{\left(z f^{\prime}(z)\right)^{\prime}}{\phi^{\prime}(z)}\right]>0
$$

for $z$ in $E$. For a fixed $\alpha$ we denote by $C_{\alpha}$ the class of $\alpha$-close-to-convex functions.

In $\$ 2$ we shall show that all $\alpha$-close-to-convex functions are close-to-convex

Received by the editors May 26, 1975 and, in revised form, September 22, 1975.

AMS (MOS) subject classifications (1970). Primary 30A32.

Key words and phrases. Alpha-starlike functions, convex functions, starlike functions, close-toconvex functions, radius of univalence. 
and $C_{\alpha} \subset C_{\beta}$ if $\alpha>\beta \geqslant 0$. It is interesting to note that $C_{\infty}$ is the class of all convex functions in $E$. In $\$ 3$ we find an integral representation formula and a few coefficient estimates for the class of $\alpha$-close-to-convex functions. In $\$ 4$ we shall define subclasses of the class of functions whose derivative has positive real part in $E$ and study some of their properties.

2. To prove our main theorem we shall require the following lemma.

LEMma 1. Let $\alpha \geqslant 0$ and $D(z)$ be a starlike function in the open unit disc $E$. Let $N(z)$ be analytic in $E$ and $N(0)=D(0)=0=N^{\prime}(0)-1=D^{\prime}(0)-1$. Then $\operatorname{Re}(N(z) / D(z))>0$ for $z$ in $E$ whenever

$$
\operatorname{Re}\left[(1-\alpha) \frac{N(z)}{D(z)}+\alpha \frac{N^{\prime}(z)}{D^{\prime}(z)}\right]>0
$$

for $z$ in $E$.

The above lemma includes as a particular case a well-known and widely used lemma of Sakaguchi [10] which corresponds to the case $\alpha=1$.

Proof. Let an analytic function $\omega(z)$ be defined in $E$ by

$$
N(z) / D(z)=(1-\omega(z)) /(1+\omega(z)) .
$$

Evidently $\omega(0)=0, \omega(z) \neq-1$ for $z$ in $E$. We shall now prove that $|\omega(z)|<1$ for $z$ in $E$. For if not, then by Jack's lemma [1] we could find $\zeta,|\zeta|<1$, such that $|\omega(\zeta)|=1$ and

$$
\zeta \omega^{\prime}(\zeta)=k \omega(\zeta)
$$

where $k \geqslant 1$.

We write

$$
\psi(z)=(1-\alpha) \frac{N(z)}{D(z)}+\alpha \frac{N^{\prime}(z)}{D^{\prime}(z)} .
$$

From (2.2), (2.3) and (2.4), we have

$$
\psi(\zeta)=\frac{1-\omega(\zeta)}{1+\omega(\zeta)}-\frac{2 \alpha k \omega(\zeta)}{(1+\omega(\zeta))^{2}} \frac{D(\zeta)}{\zeta D^{\prime}(\zeta)}
$$

with $|\omega(\zeta)|=1$. Since

$$
\operatorname{Re} \frac{1-\omega(\zeta)}{1+\omega(\zeta)}=0, \quad \operatorname{Re} \frac{D(\zeta)}{\zeta D^{\prime}(\zeta)}>0
$$

and $\omega(\zeta) /(1+\omega(\zeta))^{2}$ is real and positive, therefore $\operatorname{Re} \psi(\zeta) \leqslant 0$. This contradicts the given hypotheses that $\operatorname{Re}(\psi(z))>0$ for $z$ in $E$. Then (2.2) gives $\operatorname{Re}(N(z) / D(z))>0$ for $z$ in $E$.

On choosing $D(z)=\phi(z)$ and $N(z)=z f^{\prime}(z)$ in the above lemma, we arrive at 
TheOREM 1. Let $f(z) \in C_{\alpha}$ and $\alpha \geqslant 0$. Then $f(z)$ is close-to-convex in $E$.

TheOrem 2. Let $\alpha>\beta \geqslant 0$. Then $C_{\alpha} \subset C_{\beta}$.

Proof. If $\beta=0$, then $C_{\alpha} \subset C_{0}$, By Theorem 1. Let $\beta \neq 0$ and $f(z) \in C_{\alpha}$. Then there exists a starlike function $\phi(z)$ such that (1.2) holds. Therefore by Lemma 1,

$$
\operatorname{Re}\left(z f^{\prime}(z) / \phi(z)\right)>0
$$

for $z$ in $E$. From (1.2), (2.5) and the identity

$$
\begin{aligned}
(1-\beta) & \frac{z f^{\prime}(z)}{f(z)}+\beta \frac{\left(z f^{\prime}(z)\right)^{\prime}}{\phi^{\prime}(z)} \\
& =\frac{\beta}{\alpha}\left[\left(\frac{\alpha}{\beta}-1\right) \frac{z f^{\prime}(z)}{\phi(z)}+(1-\alpha) \frac{z f^{\prime}(z)}{\phi(z)}+\alpha \frac{\left(z f^{\prime}(z)\right)^{\prime}}{\phi^{\prime}(z)}\right],
\end{aligned}
$$

we arrive at the desired result $f(z) \in C_{\beta}$.

3. TheOREM 3 (INTEgRAL REPRESENTATION FORMUla). A function $f(z)$ is in $C_{\alpha}$ if and only if there exists a starlike function $\phi(z)$ and a function $P(z)$ which is regular and has positive real part in $E$ such that

$$
f^{\prime}(z)=\frac{1+c}{z[\phi(z)]^{c}} \int_{0}^{z}[\phi(\zeta)]^{c} \phi^{\prime}(\zeta) P(\zeta) d \zeta
$$

where $c=(1 / \alpha)-1, \alpha \neq 0$. If $\alpha=0$, then

$$
f^{\prime}(z)=(\phi(z) / z) P(z) .
$$

(Powers in (3.1) are meant as principal values.)

Proof. Let $f(z) \in C_{\alpha} \quad(\alpha>0)$. Then the quantity in brackets in (1.2) equals a function $P(z)$ with positive real part in $E$. Multiplying both sides of this equality by $\alpha^{-1}[\phi(z)]^{c} \phi^{\prime}(z)$ we obtain

$$
c z f^{\prime}(z)(\phi(z))^{c-1} \phi^{\prime}(z)+(\phi(z))^{c}\left(z f^{\prime}(z)\right)^{\prime}=(1+c)(\phi(z))^{c} \phi^{\prime}(z) P(z) .
$$

The left-hand side of (3.2) is the exact differential of $z f^{\prime}(z)(\phi(z))^{c}$. Therefore on integrating both sides of (3.2) with respect to $z$ we obtain (3.1).

Conversely, if $f(z)$ satisfies (3.1), then it is easy to see that $f(z) \in C_{\alpha}$.

On choosing $\phi(z)=z /(1-z)^{2}$ and $P(z)=(1+z) /(1-z)$ in (3.1), we obtain the following function of $C_{\alpha}$ :

$$
\begin{array}{r}
f(z)=\int_{0}^{z}(1+c) t^{-c-1}(1-t)^{2 c}\left(\int_{0}^{t} \frac{\zeta^{c}(1+\zeta)^{2}}{(1-\zeta)^{2 c+4}} d \zeta\right) d t \\
\quad(c=(1 / \alpha)-1) .
\end{array}
$$

The function (3.3) will serve as an extremal function for the next theorem.

TheOREM 4. Let $f(z)=z+a_{2} z^{2}+a_{3} z^{3}+\cdots$ be in $C_{\alpha}$. Then we have the sharp inequalities: 


$$
\left|a_{2}\right| \leqslant(2+\alpha) /(1+\alpha)
$$

$$
\left|a_{3}\right| \leqslant\left(9+23 \alpha+6 \alpha^{2}\right) /(3(1+\alpha)(1+2 \alpha)) \text {, }
$$

$$
\left|a_{4}\right| \leqslant\left(4+22 \alpha+34 \alpha^{2}+6 \alpha^{3}\right) /((1+\alpha)(1+2 \alpha)(1+3 \alpha)) .
$$

Proof. Let $f(z)=z+a_{2} z^{2}+\cdots \in C_{\alpha}$. Then there exists a starlike function $\phi(z)=z+b_{2} z^{2}+\cdots$ and a function $P(z)=1+c_{1}, z+c_{2} z^{2}$ $+\cdots$ with positive real part in $E$ such that the quantity in brackets in (1.2) equals $P(z)$. Multiplying both sides of this equality with $\phi(z) \phi^{\prime}(z)$ and then substituting power series expansions of $f(z), \phi(z)$ and $P(z)$ in it, we obtain

$$
\begin{aligned}
(1-\alpha) & \left(z+2 a_{2} z^{2}+3 a_{3} z^{3}+\cdots\right)\left(1+2 b_{2} z+3 b_{3} z^{2}+\cdots\right) \\
& +\alpha\left(z+b_{2} z^{2}+b_{3} z^{3}+\cdots\right)\left(1+4 a_{2} z+9 a_{3} z^{2}+16 a_{4} z^{3}+\cdots\right) \\
= & \left(1+c_{1} z+c_{2} z^{2}+\cdots\right)\left(z+b_{2} z^{2}+b_{3} z^{3}+\cdots\right) \\
& \cdot\left(1+2 b_{2} z+3 b_{3} z^{2}+4 b_{4} z^{3}+\cdots\right) .
\end{aligned}
$$

On comparing the coefficients of $z^{2}, z^{3}$ and $z^{4}$ on both sides of (3.7), we obtain

$$
\begin{gathered}
2(1+\alpha) a_{2}=(1+\alpha) b_{2}+c_{1}, \\
3(1+2 \alpha) a_{3}=-4 a_{2} b_{2}+(1+2 \alpha) b_{3}+3 b_{2} c_{1}+2 b_{2}^{2}+c_{2},
\end{gathered}
$$

and

$$
\begin{aligned}
4(1+3 \alpha) a_{4}= & -(6+3 \alpha) a_{3} b_{2}+(2 \alpha-6) a_{2} b_{3}+(3 \alpha-4) b_{4} \\
& +5 b_{4}+5 b_{2} b_{3}+\left(4 b_{3}+2 b_{2}^{2}\right) c_{1}+3 b_{2} c_{2}+c_{3} .
\end{aligned}
$$

Since $\operatorname{Re}(P(z))>0$ for $z$ in $E$, therefore [6]

$$
\left|c_{n}\right| \leqslant 2, \quad n=1,2,3, \ldots
$$

Again $\phi(z)$ being starlike in $E$, its coefficients satisfy the following inequalities:

$$
\left|b_{n}\right| \leqslant n, \quad n=2,3, \ldots,
$$

and

$$
\left|b_{3}-\mu b_{2}^{2}\right| \leqslant 3-4 \mu
$$

if $\mu \leqslant \frac{1}{2}$. (3.12) is well known [6] and (3.13) is due to Keogh and Merkes [2].

On using (3.11) and (3.12), (3.8) gives (3.4). On substituting for $a_{2}$ from (3.8) in (3.9), the latter becomes

(3.14) $3(1+\alpha)(1+2 \alpha) a_{3}=(1+3 \alpha) b_{2} c_{1}+(1+\alpha)(1+2 \alpha) b_{3}+(1+\alpha) c_{2}$.

On using (3.11) and (3.12), we obtain (3.5) from (3.14). Now substitute for $a_{2}$ and $a_{3}$ from (3.8) and (3.9) respectively in (3.10). Then we arrive at 


$$
\begin{aligned}
4(1+\alpha)(1+2 \alpha)(1+ & 3 \alpha) a_{4}=(1+\alpha)(1+2 \alpha)(1+3 \alpha) b_{4} \\
& +c_{1}(1+2 \alpha)(1+5 \alpha)\left[b_{3}-\frac{\alpha(1-\alpha)}{(1+2 \alpha)(1+5 \alpha)} b_{2}^{2}\right] \\
& +b_{2} c_{2}(1+\alpha)(1+5 \alpha)+c_{3}(1+\alpha)(1+2 \alpha) .
\end{aligned}
$$

Notice that $(\alpha(1-\alpha) /((1+2 \alpha)(1+5 \alpha))) \leqslant 1 / 2$. Therefore on using (3.11), (3.12) and (3.13) in (3.15), we arrive at

$$
\begin{aligned}
4(1+\alpha)(1+2 \alpha)(1 & +3 \alpha)\left|a_{4}\right| \leqslant 4(1+\alpha)(1+2 \alpha)(1+3 \alpha) \\
& +2(1+2 \alpha)(1+5 \alpha)[3-4 \alpha(1-\alpha) /(1+2 \alpha)(1+5 \alpha)] \\
& +4(1+\alpha)(1+5 \alpha)+2(1+\alpha)(1+2 \alpha) .
\end{aligned}
$$

On simplification, the above inequality reduces to (3.6).

Equality occurs in (3.4) to (3.6) for $f(z)$ given by (3.3).

It is evident that the above method cannot be easily employed for finding bounds on higher coefficients.

4. Functions whose derivative has positive real part in the unit disc. Let $\mathscr{F}_{\alpha}$ denote the class of functions $f(z)=z+a_{2} z^{2}+\cdots$ which are regular in $E$ and satisfy the condition

$$
\operatorname{Re}\left(f^{\prime}(z)+\alpha z f^{\prime \prime}(z)\right)>0
$$

for $z$ in $E$. On putting $\alpha=0$, we obtain the class $\mathscr{F}_{0}$ of functions $f(z)$ whose derivative has positive real part in $E$.

TheOREM 5. Let $f(z) \in \mathscr{F}_{\alpha}$ and $\operatorname{Re} \alpha \geqslant 0$. Then $\operatorname{Re} f^{\prime}(z)>0$ for $z$ in $E$.

Proof. The proof follows on choosing $N(z)=z f^{\prime}(z)$ and $D(z)=z$ in Lemma 1.

It is easy to see that $\mathscr{F}_{\alpha} \subset \mathscr{F}_{\beta}$ if $\alpha>\beta \geqslant 0$ and that for each fixed $\alpha>0$ the functions of the class $\mathscr{F}_{\alpha}$ are uniformly bounded in $E$. If $0 \leqslant \lambda \leqslant 1$ and $f(z)$ and $g(z)$ be in $\mathscr{F}_{\alpha}$, then $F(z)=(1-\lambda) f(z)+\lambda g(z)$ is also in $\mathscr{F}_{\alpha}$. Functions of the class $\mathscr{F}_{\alpha}$ are obtained on taking the Hadamard product of the convex function

$$
k(z)=\frac{1}{\alpha} z^{1-1 / \alpha} \int_{0}^{z} \frac{\zeta^{1 / \alpha-1}}{1-\zeta} d \zeta
$$

with $\int_{0}^{z} P(t) d t$, where $P(z)$ is an arbitrary function with positive real part in $E$. (For definition of $f * g$, the Hadamard product of $f$ and $g$, see [9].)

5. In this section we shall define a class of functions which is closely related to the class $\mathscr{F}_{\alpha}$. We say a regular function $f(z)$ is in $\mathcal{G}_{\alpha}$ if $f(z)$ is regular in $E, f(0)=0=f^{\prime}(0)-1$, and if it satisfies the condition

$$
\operatorname{Re}\left[(1-\alpha) f(z) / z+\alpha f^{\prime}(z)\right]>0
$$

for $z$ in $E$ and for some $\alpha \geqslant 0$. Then $f(z) \in \mathscr{F}_{\alpha}$ if and only if $z f^{\prime}(z) \in \mathcal{G}_{\alpha}$.

From Lemma 1 it follows that if $f(z) \in \mathcal{G}_{\alpha}$, then $\operatorname{Re}(f(z) / z)>0$ for $z$ in 
$E$. On combining this fact with (5.1) we see that $f(z) \in \mathcal{G}_{\alpha}$ gives $\operatorname{Re}\left(f^{\prime}(z)\right)$ $>0$ for $z$ in $E$ provided $\alpha \geqslant 1$. Functions of $\mathcal{G}_{\alpha}$ are univalent in $E$ if $\alpha \geqslant 1$ [8]. MacGregor [3] showed that the functions of the class $\mathcal{G}_{0}$ are univalent in $|z|<\sqrt{ } 2-1$. In Theorem 6 below we shall find sharp radius of univalence of $\mathcal{G}_{\alpha}, 0<\alpha<1$.

We shall require the following lemma in the proof of the next theorem.

LEMMA 2. Let $P(z)$ and $Q(z)$ be regular in $E, P(0)=Q(0)=1, \operatorname{Re} P(z)>0$ and $\operatorname{Re} Q(z)>1 / 2$ for $z$ in $E$. Then $\operatorname{Re}(P(z) * Q(z))>0$ for $z$ in $E$.

The above lemma is equivalent to a lemma of Nehari and Netanyahu [7]. The following lemma is a simple consequence of the above lemma.

LEMMA $2^{\prime}$. Let $P(z)$ and $Q(z)$ be regular in $E, P(0)=Q(0)=1, \operatorname{Re} P(z)$ $>0$ for $z$ in $E$, and $\operatorname{Re} Q(z)>1 / 2$ for $z$ in $|z|<\rho(0<\rho \leqslant 1)$. Then

$$
\operatorname{Re}(P(z) * Q(z))>0
$$

for $z$ in $|z|<\rho$.

THEOREM 6. Let $0<\alpha<1$ and $f(z) \in \mathcal{G}_{\alpha}$. Then $f(z)$ is univalent and satisfies $\operatorname{Re} f^{\prime}(z)>0$ in $|z|<r_{0}$, where $r_{0}$ is the smallest positive root of the equation

$$
\frac{2 c-1-r}{1+r}-2 c(c-1) \int_{0}^{1} \frac{t^{c-1}}{1+t r} d t=0
$$

where $c=1 / \alpha$. This result is sharp.

Proof. Let $f(z) \in \mathcal{G}_{\alpha}$. Then it is easy to see that there exists a function $P(z)$ which is regular in $E$ and satisfies the conditions $P(0)=1$ and $\operatorname{Re} P(z)>0$ for $z$ in $E$ such that

$$
f(z)=z P(z) * k(z)
$$

where $k(z)$ is defined by (4.2). On differentiating both sides of (5.3) with respect to $z$ we obtain

$$
f^{\prime}(z)=P(z) * g(z)
$$

where

$$
g(z)=k^{\prime}(z)=\frac{c}{1-z}-c(c-1) z^{-c} \int_{0}^{z} \frac{\zeta^{c-1}}{1-\zeta} d \zeta
$$

Powers in (5.4) are meant as principal values. The function $g(z)$ is regular for $z$ in $E, g(0)=1$ and

$$
2 g(z)-1=\frac{1+z}{1-z}+2 c(c-1) \int_{0}^{1} t^{c-1}\left[\frac{1}{1-z}-\frac{1}{1-t z}\right] d t
$$

It is easy to see that for $|z|=r, 0 \leqslant r<1$, 


$$
\begin{aligned}
2 \operatorname{Re}(g(z))-1 & \geqslant \frac{1-r}{1+r}-2 c(c-1) \int_{0}^{1} \frac{t^{c-1}(1-t) r}{(1+r)(1+t r)} d t \\
& =\frac{2 c-1-r}{1+r}-2 c(c-1) \int_{0}^{1} \frac{t^{c-1}}{1+t r} d t .
\end{aligned}
$$

Therefore $\operatorname{Re}(g(z))>1 / 2$ in $|z|<r_{0}$ where $r_{0}$ is the smallest positive root of (5.2). The theorem now follows from Lemma $2^{\prime}$.

The function

$$
f_{0}(z)=2 k(z)-1=z+\sum_{n=2}^{\infty} \frac{2 c z^{n}}{c-1+n}
$$

is obtained on putting $P(z)=(1+z) /(1-z)$ in (5.3). Therefore $f_{0}(z)$ is in $\mathcal{S}_{\alpha}$. For this function $f_{0}^{\prime}\left(-r_{0}\right)=0$. This shows that the above result is sharp.

The author is grateful to Professor Vikramaditya Singh for his encouragement during the prepration of this paper.

\section{REFERENCES}

1. I. S. Jack, Functions starlike and convex of order $\alpha$, J. London Math. Soc. (2) 3 (1971), 469-474. MR 43 \#7611.

2. F. R. Keogh and E. P. Merkes, A coefficient inequality for certain classes of analytic functions, Proc. Amer. Math. Soc. 20 (1969), 8-12. MR 38 \# 1249.

3. T. H. MacGregor, Functions whose derivative has a positive real part, Trans. Amer. Math. Soc. 104 (1962), 532-537. MR 25 \#4090.

4. P. T. Mocanu, Une propriété de convexité généralisée dans la théorie de la représentation conforme, Mathematica (Cluj) 11 (34) (1969), 127-133. MR 42 \#881.

5. S. S. Miller, P. T. Mocanu and M. O. Reade, All $\alpha$-convex functions are univalent and starlike, Proc. Amer. Math. Soc. 37 (1973), 553-554. MR 47 \#2044.

6. Z. Nehari, Conformal mapping, McGraw-Hill, New York, 1952. MR 13, 640.

7. Z. Nehari and E. Netanyahu, On the coefficients of meromorphic schlicht functions, Proc. Amer. Math. Soc. 8 (1957), 15-23. MR 18, 648.

8. K. Noshiro, On the theory of schlicht functions, J. Fac. Sci. Hokkaido Univ. (1) 2 (1934/35), 129-155.

9. St. Ruscheweyh and T. Sheil-Small, Hadamard products of schlicht functions and the PólyaSchoenberg conjecture, Comment. Math. Helv 48 (1973), 119-135. MR 48 \#6393.

10. K. Sakaguchi, On a certain univalent mapping, J. Math. Soc. Japan 11 (1959), 72-75. MR 21 \# 5734.

Department of Physics, Punjabi University, Patiala-147002, India 